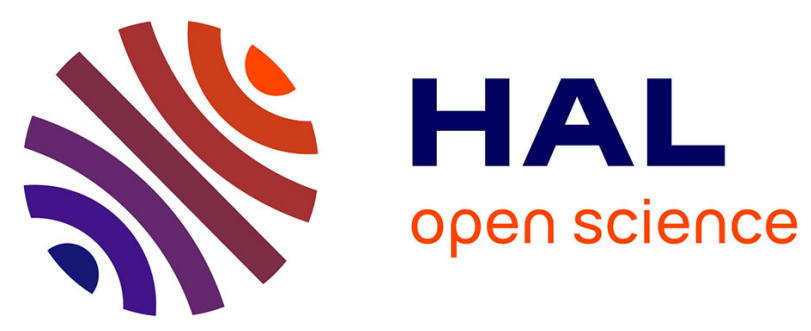

\title{
Enhancing photocatalytic performance and solar absorption by schottky nanodiodes heterojunctions in mechanically resilient palladium coated $\mathrm{TiO} 2 / \mathrm{Si}$ nanopillars by atomic layer deposition
}

Emerson Coy, Katarzyna Siuzdak, Mykola Pavlenko, Karol Zalęski, Octavio Graniel, Marcin Ziólek, Sébastien Balme, Philippe Miele, Matthieu Weber, Mikhael Bechelany, et al.

\section{- To cite this version:}

Emerson Coy, Katarzyna Siuzdak, Mykola Pavlenko, Karol Zalęski, Octavio Graniel, et al.. Enhancing photocatalytic performance and solar absorption by schottky nanodiodes heterojunctions in mechanically resilient palladium coated $\mathrm{TiO} 2 / \mathrm{Si}$ nanopillars by atomic layer deposition. Chemical Engineering Journal, 2020, 392, pp.123702. 10.1016/j.cej.2019.123702 hal-02537086

\author{
HAL Id: hal-02537086 \\ https://hal.science/hal-02537086
}

Submitted on 31 May 2021

HAL is a multi-disciplinary open access archive for the deposit and dissemination of scientific research documents, whether they are published or not. The documents may come from teaching and research institutions in France or abroad, or from public or private research centers.
L'archive ouverte pluridisciplinaire HAL, est destinée au dépôt et à la diffusion de documents scientifiques de niveau recherche, publiés ou non, émanant des établissements d'enseignement et de recherche français ou étrangers, des laboratoires publics ou privés. 


\title{
Enhancing Photocatalytic Performance and Solar Absorption by Schottky Nanodiodes Heterojunctions in Mechanically Resilient Palladium Coated $\mathrm{TiO}_{2} / \mathrm{Si}$ Nanopillars by Atomic Layer Deposition
}

Emerson Coy $^{1 *}$, Katarzyna Siuzdak ${ }^{2}$, Mykola Pavlenko ${ }^{1}$, Karol Załęski ${ }^{1}$, Octavio Graniel ${ }^{3}$, Marcin Ziółek ${ }^{4}$, Sebastien Balme ${ }^{3}$, Philippe Miele ${ }^{3}$, Matthieu Weber ${ }^{3}$, Mikhael Bechelany ${ }^{3 *}$, Igor Iatsunskyi ${ }^{*}$

1. NanoBioMedical Centre, Adam Mickiewicz University, Wszechnicy Piastowskiej 3, 61-614, Poznan, Poland

2. Centre of Laser and Plasma Engineering, The Szewalski Institute of Fluid-Flow Machinery, Fiszera 14 Str., 80-231 Gdansk, Poland

3. Institut Européen des Membranes, IEM-UMR 5635, ENSCM, CNRS, Univ Montpellier, Montpellier, France

4. Faculty of Physics, Adam Mickiewicz University in Poznań, Umultowska 85, 61-614 Poznań, Poland.

Corresponding authors*: coyeme@amu.edu.pl, mikhael.bechelany@umontpellier.fr, igoyat@amu.edu.pl.

Key Words: Photocatalytic; Solar Heat Conversion; Atomic Layer Deposition; Nanopillars; Photodegradation;

\begin{abstract}
The development of highly efficient photocatalytic materials and composites has been one of the main goals in the energy and environmental fields. To this date, however, the efficiency and stability of photocatalytic materials are still very low. This work shows a novel strategy of Pd coated $\mathrm{TiO}_{2} / \mathrm{Si}$ nanopillars produced by atomic layer deposition with a wide light absorption window. The structures show significantly improved performance due to the synergistic effect of $\mathrm{Pd}$ decorated $\mathrm{TiO}_{2} / \mathrm{Si}$ nanopillars, and the cooperative effect between Hot and Photoexcited electrons and Schottky nanojunctions. The performance of the composites is evaluated, the enhancement mechanism is explained and the advantages of the rational design of these materials are discussed.
\end{abstract}

\section{Introduction}

Solar water splitting mediated by photocatalytically active nanostructures is one of the most promising strategies toward the potential implementation of hydrogen energy in the future. ${ }^{1-3}$ Electrolysis, the production of molecular hydrogen by electrical means from aqueous solutions, produces a high yield of usable hydrogen at a rather energetically expensive cost, making it an unpractical solution for a hydrogen-based economy. ${ }^{4}$ On the other hand, photoactive materials could be used to harness our plentiful solar radiation to generate electron-rich centres for catalytic production of hydrogen or combined remediation strategies. ${ }^{5}$ Nowadays, several architectures have been proposed to improve the absorption of sunlight in various materials to boost the 
efficiency of hydrogen producing devices. A common strategy found throughout the literature aims to broaden the absorption window of photoactive materials, ${ }^{5}$ which typically resides below the visible wavelength of light (UV). ${ }^{6}$ This broadening attempt is due to the rather large bandgap of common light-harvesting materials like Titanium oxide $\left(\mathrm{TiO}_{2}\right)$ and Zinc oxide $(\mathrm{ZnO}){ }^{7}$ that limits their conversion efficiency.

Another strategy to improve the photoactivity of these materials is to control their morphology and exploit confinement or nanometric effects. ${ }^{6,8,9}$ During the last few years, several groups (including our own) have been actively working on silicon (Si) nanopillars (NPls) ${ }^{6,10}$ due to their well-established commercial and industrial applicability, availability, and the relatively small bandgap $\left(\mathrm{E}_{\mathrm{g}}=1.1 \mathrm{eV}\right)$ of silicon. Moreover, Si-NPls are quite attractive structures that have shown wider absorption and enhanced performance due to the clear increment of their active surface. ${ }^{11,12}$ However, as photoanode, bare Si suffers from fast degradation and corrosion in aqueous environments, which dramatically limits its operation time ${ }^{13}$ Several strategies based on the introduction of protective coatings to hinder surface passivation have been proposed to extend the lifetime of Si based photoanodes ${ }^{14,15}$ Additional studies have shown the advantages of $\mathrm{TiO}_{2}$ or $\mathrm{ZnO}$ coatings on the performance of Si-NPls, ${ }^{16-18}$ due to their optical tunability, ${ }^{6}$ mechanical reinforcement, ${ }^{10,19}$ and high photoactive performance. ${ }^{6,10}$

Recently, photoactive junctions of precious metals and semiconducting materials have shown enhanced carrier transport capabilities of photogenerated electrons which could provide a more efficient catalytic performance for water splitting ${ }^{20-26}$. In principle, photoactive junctions between precious metals and semiconducting oxides are more efficient when contact areas are maximized. Thus, nanocomposite-like structures are preferred over heterostructures, although they have shown important results in photocatalysis ${ }^{27}$. Nevertheless, the maximal efficiency of photojunctions on nanostructured surfaces, such NPls, is rather demanding since the anchoring of the particles is limited by physical techniques, ${ }^{28,29}$ or by the anchoring point of thermal decomposition methods. ${ }^{30}$ Atomic Layer deposition (ALD) is a well-stablished technique for the synthesis of thin films and nanoparticles, as these nanomaterials can be prepared with an extreme control over their dimensions ${ }^{31}$, excellent uniformity over the substrate, and conformality on nanometer-sized ${ }^{31-33}$. Recently, we developed and tested an ALD route for the deposition of a highly conformal Pd layer, in which the nucleation and surface coverage can be maximized while 
retaining anchoring strength. ${ }^{34-36}$ The efficiency of this method was further tested in $\mathrm{Pd} / \mathrm{TiO}_{2}$ nanotubes junction for environmental applications. ${ }^{37}$

On the other hand, several groups have shown the importance of photothermal water vaporization as a strategy for remediation and photocatalysis. ${ }^{38,39}$ One of the advantages of this methodology is the direct conversion of solar energy into chemical energy due to the efficient light-harvesting, ${ }^{38}$ heat confinement, ${ }^{40}$ and no need to transport or inject electrons into the system. ${ }^{38,39}$ As expected, one of the main strategies to induce full solar spectrum absorption is nanostructuring and/or nanopatterning of surfaces to reduce their optical reflection. ${ }^{41}$ Additionally, metals have shown outstanding performance as solar absorbers, due to rather large excitation, plasmonic effects, ${ }^{42}$ and heat transport properties. ${ }^{43}$ For instance, palladium (Pd) has been used as main collector of solar energy in steam generation applications with outstanding results. ${ }^{40}$ Similarly, the cooperative nature of $\mathrm{Au} @ \mathrm{TiO}_{2}$ junctions for photocatalysis and solar evaporation in remediation strategies has also been shown. ${ }^{44}$

In the present work, we investigate the synergistic effect of $\mathrm{Pd}$ decorated $\mathrm{TiO}_{2} / \mathrm{Si}-\mathrm{NPl}$ for photocatalytic applications. The effective decoration of $\mathrm{Pd}$ by $\mathrm{ALD}$ will be demonstrated. Additionally the capability of this route to enable and enhance the catalytic activity to the asprepared structures will be discussed. The improvement of catalytic performance of the nanostrucutres will be followed by an additional enhancement in heat absorption by the Pd layer. A comprehensive physico-chemical characterization of the pillars will be presented by means of X-ray diffraction (XRD), Scanning Electron Microscopy (SEM), Transmission Electron Microscopy (TEM), Energy Dispersive Spectroscopy (EDX), Nanoindendation, Ultraviolet Visible spectroscopy (UV-Vis). The synergistic effect originating from the noble metal and the ordered substrate will be revealed by Electro Impedance Spectroscopy (EIS), Chronoamperometry, and cyclic voltammetry (CV). Also, we will show that water evaporation rates are boosted, and that the general cooperative performance of nanostructures, although complex in nature, could be explained by a combined OER/HER mechanism. The general applicability of the photocatalytic properties of $\mathrm{TiO}_{2} / \mathrm{Si}$ and solar absorption of the $\mathrm{Pd} / \mathrm{TiO}_{2}$ junctions are evaluated and allow for a further strategy to enhance the performance of photoactive junctions exploiting a broader range of the light spectra.

\section{Results and Discussion}




\section{Structural and Optical Characterization}

The structure and morphology of Si-NPls were investigated primarily by SEM and XRD. Figure 1. After the Metal-assisted chemical etching (MACE) procedure, pillars are predominantly placed in an hexagonal matrix (Figure S1), with an average diameter of $450 \pm 50 \mathrm{~nm}$, length of $3.0 \pm 0.2 \mu \mathrm{m}$, and base separation of $200 \pm 50 \mathrm{~nm}$ (Figure 1a,b). After the coating process of $\mathrm{TiO}_{2}$ by ALD, a layer of $25 \pm 3 \mathrm{~nm}$ was observed, and the general morphology of the Si pillars was unaffected. The phase of the layers was investigated by GI-XRD(Figure 1c). The patterns show two main peaks at around $\sim 25^{\circ}$ and $\sim 47^{\circ}$ 2theta, corresponding to the (101) and (200) peaks

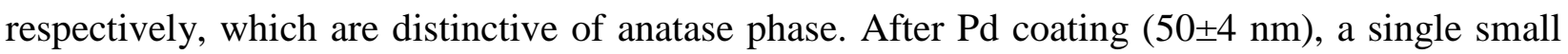
peak at around $\sim 40^{\circ}$ 2theta is observed, corresponding to the (111) and confirming the crystallinity of both $\mathrm{TiO}_{2}$ and $\mathrm{Pd}$ coating processes. TEM and EDX mappings, Figure 1d, show the core shell structure of the $\mathrm{Pd} / \mathrm{TiO}_{2} / \mathrm{Si}$ pillars with well-defined thickness and distribution, additionally, independent maps are shown in Figure S2. The high resolution TEM images show the conformal structure of the coatings. Moreover, the FFT patterns shown in the inset confirm the GI-XRD results by showing $\mathrm{TiO}_{2}$ anatase and $\mathrm{Pd}$ crystalline phases.. It is important to notice the conformal growth of the $\mathrm{Pd}$ layer over the $\mathrm{TiO}_{2}$ with a well-defined interface and rather short mixing.
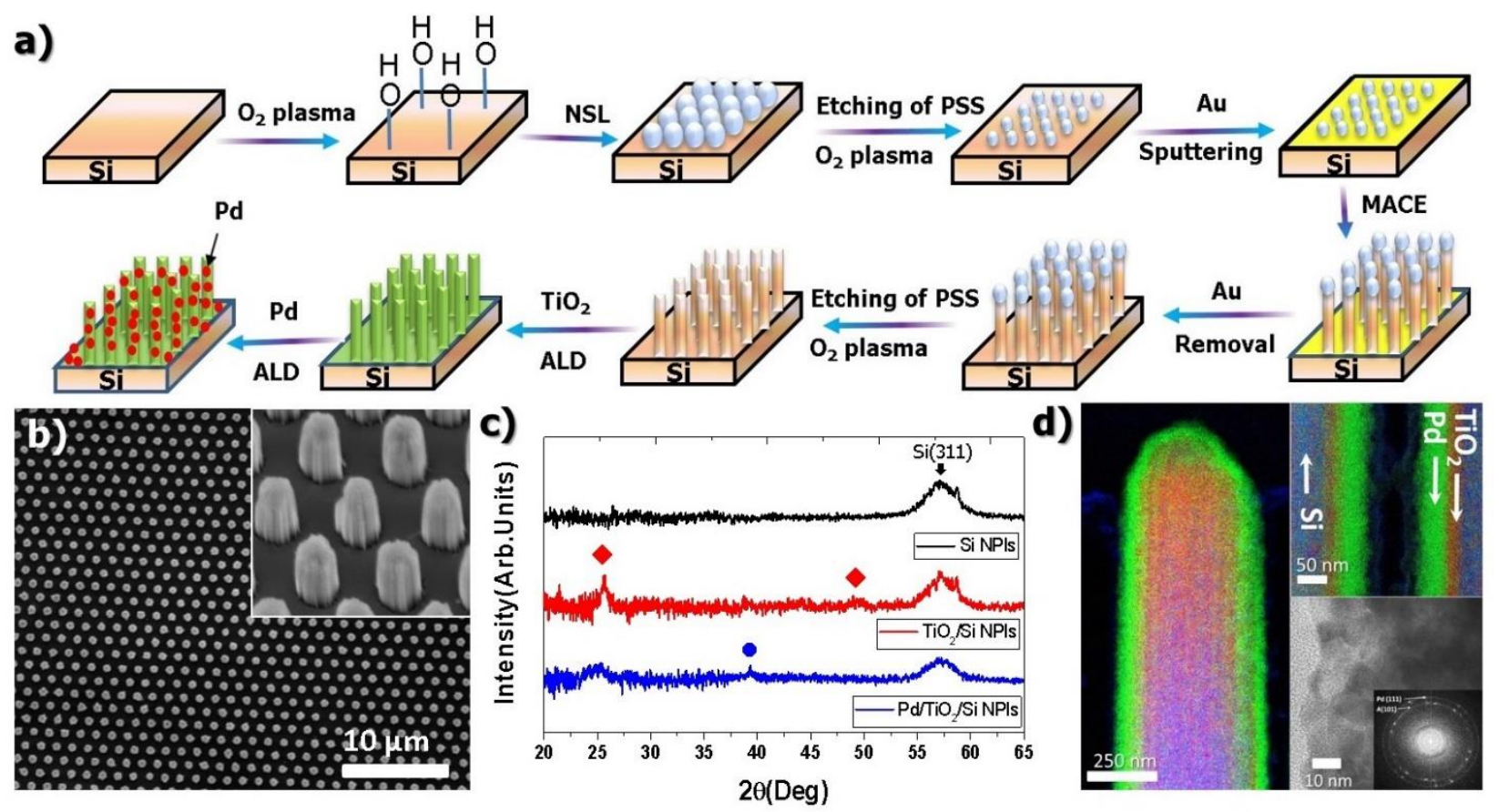

Figure 1: a) Schematic representation of the procedure for sample preparation of Si-NPls, the ALD coating by $\mathrm{TiO}_{2}$ and $\mathrm{Pd}$. b) SEM micrographs of large area ordered pillars prepared by 
Metal-assisted chemical etching (MACE). Inset shows close view of the top of the pillars. c) GIXRD images taken for bare pillars, $\mathrm{TiO}_{2}$ coated and Pd coated, showing the distinctive peaks for Anatase $\mathrm{TiO}_{2}(\diamond)$ and $\mathrm{Pd}(\bullet)$. d) STEM-EDX images showing the elemental mapping of the core shell pillars. The sample is s FIB cross-section, green is Palladium, red shows Titanium and blue the silicon area. Inset show the HR-TEM images with FFT indexed peaks of $\mathrm{TiO}_{2}$ and $\mathrm{Pd}$.

Previously we investigated the mechanical enhancement of $\mathrm{TiO}_{2}$ coated Si-NPls. ${ }^{10}$ Our results showed a considerable enhancement on mechanical resilience of the structures due to crystallization of $\mathrm{TiO}_{2}$ nanocrystals on the superficial pores of the Si-NPls. The effect of the Pd conformal coating on the $\mathrm{TiO}_{2} / \mathrm{Si}-\mathrm{NPls}$ is shown in Figure 2a. The low load regime shows the presence of a big plastoelastic contribution after Pd coating. Although this behaviour is observed in metals and $\mathrm{Pd}$ is a relative soft metal $(121 \mathrm{GPa})$, it remains harder than gold (78 Gpa). Additionally, nanoindentation experiments have shown to have strong dependency on porosity, ${ }^{45}$ (especially for $\mathrm{Si}$ ) allowing to calculate the average porosity of the Si-NPls. The average porosity of the as-prepared samples was calculated to be in the range of 70-80\% according to the load $v s$ displacement experiments. These results provided a Young's Modulus of 3-5 GPa (Figure S3). After $\mathrm{TiO}_{2}$ and further inclusion of $\mathrm{Pd}$, mechanical properties are enhanced. As reported before, the inclusion of $\mathrm{TiO}_{2}$ by ALD fills the porous superficial layers of the pillars and crystallizes in the anatase phase (Figure 1c) thus providing extra mechanical resilience towards deformation (Figure 2a). Moreover, after Pd covering, the samples show a larger plastic deformation than the one observed in $\mathrm{TiO}_{2} / \mathrm{Si}$ and $\mathrm{Pd}$ coated samples. However, the increased plastic deformation provides resilience of the conformal Pd coating by allowing the atoms to move away from the indenting pressure point (in the so called pile-up effect) without breaking the nanocomposite. This effect contributes to the further sealing of the surface and prevents failure of the surface by cracking at low loads. Overall, the mechanical changes in the pillars point show enhancement over the as-prepared Si-NPls.

Optical properties (absorbance and reflectance) play a crucial role for the photo-driven processes. They are reported in Figure $2 b$ and $c$. The diffuse reflectance measurements show the low dispersion of light of the Pd coated samples, which is attributed to the nanopillar and nanocomposite structure of the films. The absorption was calculated with the Kubelka-Munk model. ${ }^{46}$ Absorption spectra for the samples are presented in Figure 2c (Figure S4 for polished $\mathrm{Si}$ ). As expected, the $\mathrm{TiO}_{2}$ coated samples show an improved absorption below $450 \mathrm{~nm}$ due to its 
known optical bandgap. Furthermore, the absorption of the samples coated with Pd shows not only a 2-fold increment in the UV region, when compared with $\mathrm{TiO}_{2}$, but also an important increment in the visible region. The boost is considerably, considering the no absorbance of $\mathrm{Si}$ and $\mathrm{TiO}_{2} / \mathrm{Si}$ samples, additionally, it is important to remark that the increment of the visible region of $\mathrm{Pd}$, correspond to $60 \%$ of the $\mathrm{UV}$ region absorption of $\mathrm{TiO}_{2}\left(\mathrm{~A}-\mathrm{TiO}_{2}(<450 \mathrm{~nm})=7.1\right.$ and $\mathrm{A}$ $\left.\mathrm{Pd} / \mathrm{TiO}_{2(>650 \mathrm{~nm})}=4.3\right)$. These changes show a clear enhancement in both UV and IR absorption induced by $\mathrm{Pd}$ coated samples. This enhancement promises the enhanced photoactivity of the produced nanocomposites.
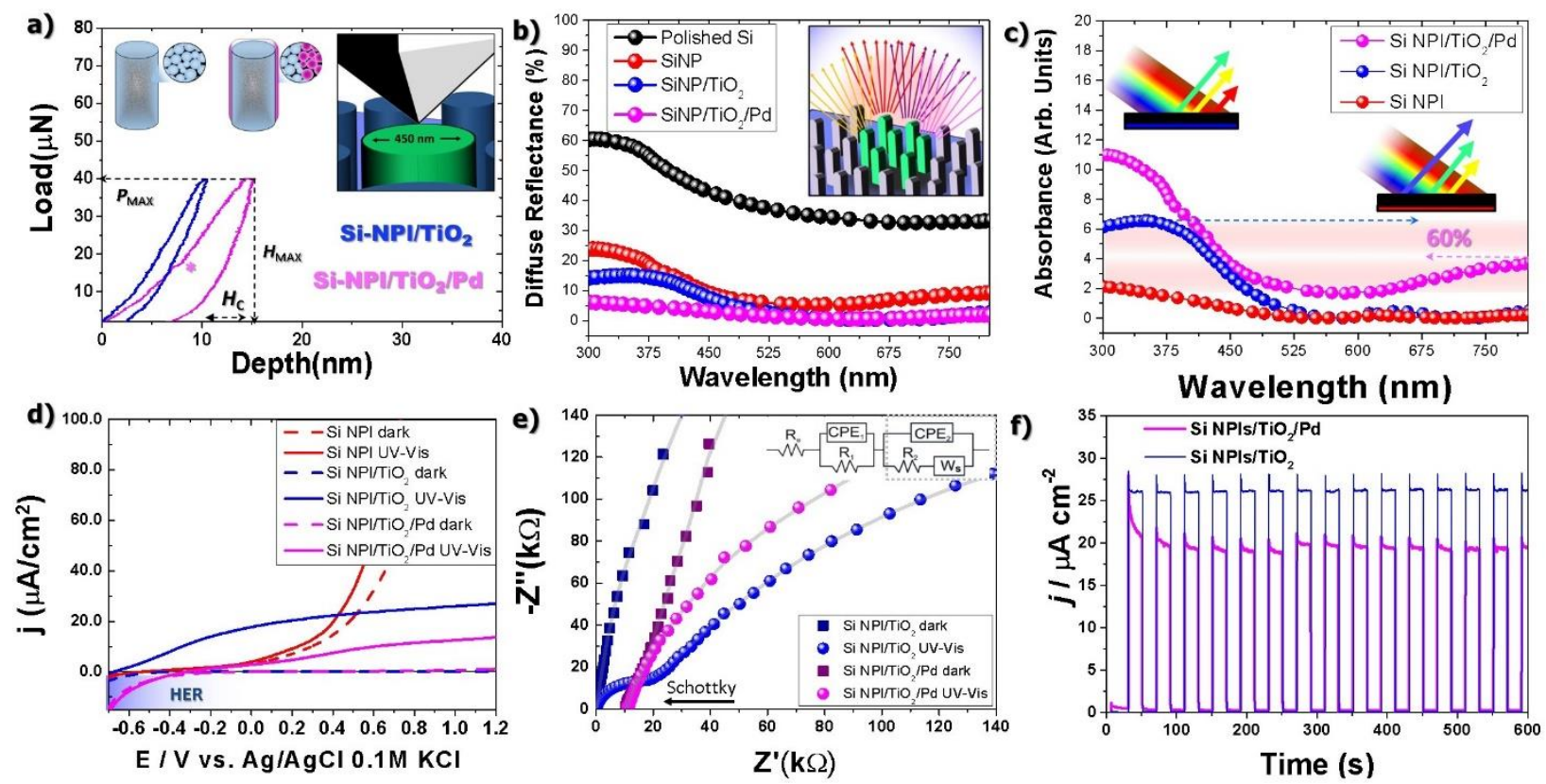

Figure 2: a) low-load indentation experiments on single pillars, $\mathrm{H}_{\max }, \mathrm{P}_{\max }$ and $\mathrm{Hc}$ show the maximum penetration, maximum load and residual imprint respectively. Insets show diagrams of the structure and indentation experiments b) Diffusive reflectance spectra of the samples c) Absorbance spectra of the prepared samples, insets show schematics of the absorption. Dashed lines are a guide to eye pointing to the IR enhanced response that corresponds to $\sim 60 \%$ of the UV absorption of bare $\mathrm{TiO}_{2}$ samples. d) Linear voltammetry curves recorded for all the samples, dashed lines correspond to dark conditions while full lines to illuminated conditions. Blue shaded region corresponds to the negative potentials where hydrogen evolution reaction takes place. e) Nyquist plots of the $\mathrm{TiO}_{2}$ and $\mathrm{Pd} / \mathrm{TiO}_{2}$ coated samples. Lines shows the response in illuminated conditions (@) and in dark conditions ( $(\boldsymbol{\square})$. Gray faded lines are the fitted curves. Inset shows the circuit model used for fitting the data. f) Chronoamperometric response of $\mathrm{TiO}_{2}$ and $\mathrm{Pd} / \mathrm{TiO}_{2}$ samples during multiple light on/off cycles collected at $+1 \mathrm{~V}$ vs. ag/AgCl/0.1M KCl.

Electrochemical and heat absorption performance 
In order to assess the efficiency of the $\mathrm{Pd} / \mathrm{TiO}_{2} / \mathrm{Si}$ structures, photoelectrochemical studies were performed. First, current density $v s$ applied voltage were recorded and are shown in Figure 2d. It is important to remark that $\mathrm{TiO}_{2} / \mathrm{Si}$ sample exhibits an important increment on photocurrent under the UV-vis light experimental conditions, while showing negligible response under dark conditions. Although the bare Si-NPls already show certain efficiency under dark conditions, these results present the proper conformal covering of the $\mathrm{TiO}_{2}$ layer on the Si-NPs. Additionally, for the Pd coated sample, no response or any other feature in dark conditions was observed, which follows a similar behaviour as the one for bare Si-NPls. It is worth to underline that the Pd inclusion, although providing a smaller photocurrent than $\mathrm{TiO}_{2}$ coated samples $(\sim 80 \%)$, also activates the negative potential region of the plots, which correspond to the hydrogen evolution reaction (HER) section of the voltamperogram shown in Figure 2d. The presence of HER is tied to the known efficiency of Pd towards hydrogen production as part of the Platinum like noble metals. ${ }^{47}$ Figure 2e shows the Nyquist plots of both the $\mathrm{TiO}_{2} / \mathrm{Si}-\mathrm{NPls}$ coated samples and $\mathrm{Pd} / \mathrm{TiO}_{2} / \mathrm{Si}-\mathrm{NlPs}$. The fitted model includes two contributions for the under illumination $\mathrm{TiO}_{2} / \mathrm{Si}-$ NPls while a single contribution is modelled for the rest. In these plots, the high frequency regime (small semicircle near the Shottky) is visible for illuminated conditions, while these contributions are not observed for dark or Pd coated samples. This observation points to a rather small resistivity of $\mathrm{TiO}_{2}$ toward the high mobility of carriers in $\mathrm{TiO}_{2}$ under illumination, which is expected for such an interface like this. However, the large and single semicircle of the Pd coated samples, coupled with the Schottky contact, suggests a lower performance of the samples as well as shielding of the photo-active centres of the film. Nevertheless, the visible changes in the EIS under illumination coupled with the chronoamperometry results, shown in Figure $2 \mathrm{f}$, which unequivocally demonstrates a rather low shielding of the photoactive centres, a $20 \%$ decrement in efficiency by the Pd coating and a rather stable response (other samples in Figure S5).
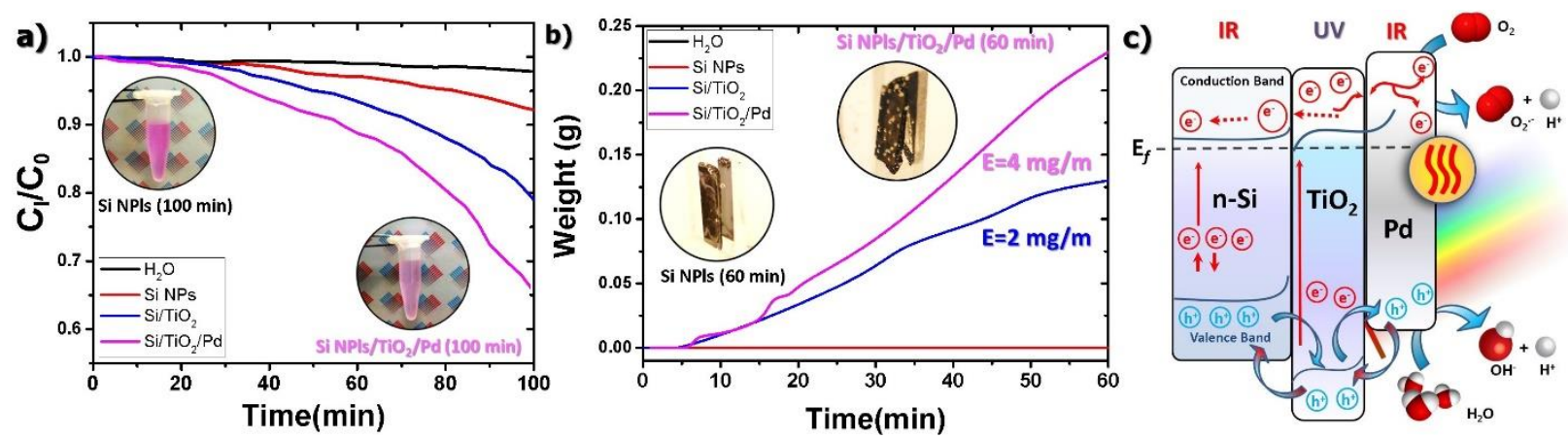
Figure 3: a) Degradation studies of Rhodamine B under UV illumination. Inset shows the pre and post decolorized solution b) Water evaporation efficiency under solar simulators conditions, inset show the quick covering of bubbles of the Pd sample over the as prepared Si-NPls. c) Shows the proposed mechanism in the cooperative degradation and photocatalysis of rhodamine b under sun-simulated conditions.

Although OER results, shown in Figure 2, suggest a decrease in performance by the Pd coating (Figure 2f), the complementary tests shown in Figure $3 \mathrm{a}$ show an enhancement photodegradation. In the typical photodegradation tests, the samples are used for the photo decomposition of rhodamine $\mathrm{B}$ in aqueous solutions ${ }^{48,49}$ The samples covered with Pd show faster decolourisation than $\mathrm{TiO}_{2} / \mathrm{Si}-\mathrm{NPls}$ and bare Si-NPls. Although the final decomposition of the Rhodamine B was not tested and the efficiency of our materials does not compete with powdered materials ${ }^{48,49}$, the experiments show a clear indication of difference in initiation of the degradation process, which is definitely enhanced by the Pd coated samples over $\mathrm{TiO}_{2} / \mathrm{Si}-\mathrm{NPls}$. Additionally, the results shown in Figure 2c suggests that a higher absorption of UV light is occurring on Pd-coated samples, while also displaying absorption on the IR region. Therefore, water evaporation rate experiments were conducted as presented in Figure $3 \mathrm{~b}$. We would like to highlight that these experiments were performed using full solar spectrum covering UV and visible range. The water evaporation results show the rapid generation of bubbles and weight loss due to heat transfer. The absorption of light, both in UV and IR clearly shows a boost of the structures towards evaporation, which opens several questions towards the applicability and mechanism of these effects.

\section{Mechanism and Origin of the enhancement}

In order to understand the origin of the enhancement in photodegradation and heat absorption of the nanostructures, several aspects need to be addressed. It has been previously shown that heat can improve the photodegradation rate of rhodamine, especially for nanoparticle-based materials. ${ }^{50}$ However, this enhancement effect has a limit since the high temperature tends to increase charge recombination and thus reducing efficiency. ${ }^{51}$ Figure S6 shows the temperature recorded during the water evaporation experiment and no considerable changes on water temperature were recorded. This shows that, although heat transfer is expected, this is not the 
main factor responsible for the improvement. A plausible explanation is the synergistic effect between the hot-electron and carrier movements occurring at the semiconducting interfaces. ${ }^{52}$ Simplifying the whole mechanism and ignoring the contribution of $\mathrm{Pd}$, UV light should be absorbed by the $\mathrm{TiO}_{2}$ layer, exciting the electrons from the valence to the conduction band and transferring them to the $n$-type $\mathrm{Si}$ nanopillars. Photogenerated holes are then gathered at the surface driving the OER. On the other hand, considering the case in which only $\mathrm{Pd} / \mathrm{TiO}_{2}$ heterojunctions are formed, the UV light would again generate electrons, which could be then transferred to the Pd surface and drive the $\mathrm{HER}^{53}$ or take part in the degradation of pollutants. ${ }^{37}$ However, it is safe to say that in our case, , when the electron transfer is happening between $\mathrm{Pd} / \mathrm{TiO}_{2} / \mathrm{Si}$, the electron transfer seems contradictory.

$\mathrm{Pd} / \mathrm{TiO}_{2}$ junctions are commonly used as sensing platforms of hydrogen due to the schottky barrier formed between $\mathrm{Pd}$ and $\mathrm{TiO}_{2}{ }^{54} \mathrm{Pd}$ is often used due to its remarkable sensitivity towards hydrogen, which is related to changes in its work function and dielectric behaviour in the presence of small quantities of hydrogen. ${ }^{55}$ This behaviour prompts the question: why is there a limited transfer of electrons within $\mathrm{Pd} / \mathrm{TiO}_{2} / \mathrm{Si}$ junctions resulting from the $\mathrm{Pd} / \mathrm{TiO}_{2}$ interface? The general barrier of $\mathrm{Pd} / \mathrm{TiO}_{2}$ has an energy of $0.85 \mathrm{eV}$ and the electron flow is established from $\mathrm{TiO}_{2}$ towards $\mathrm{Pd}$ since electrons are confined to the metal nanoparticles in these nanodiodes. ${ }^{56}$ However, the exposition to visible light and the presence of $n$-type Si makes the understanding of the electron flow difficult, which gets more complicated due to the surface Plasmon resonance, hot-electron transfer in $\mathrm{Pd}$, and direct band-gap excitation by IR wavelength in $\mathrm{Si}^{57}$

One partial solution is the case in which both HER and OER can both potentially take place. Under UV irradiation, the light is absorbed by $\mathrm{TiO}_{2}$, generating an overpopulation of electron in the conduction band, these electrons are then quickly transferred to the $n$-type silicon pillars, leading the electron flow "inwards" between $\mathrm{TiO}_{2}$ and $n$-type silicon. Additionally, the electron transfer generates a charge imbalance at the $\mathrm{Pd} / \mathrm{TiO}_{2}$ interface, that allows holes to be collected at the surface Pd surface (OER). This case provides the perfect environment for the production of super oxide anion radicals, such as $\mathrm{O}_{2}{ }^{-}$and $\mathrm{OH}^{-}$as reported in several experimental studies, for $\mathrm{Pd}^{21}, \mathrm{TiO}_{2}{ }^{58}, \mathrm{Metal} / \mathrm{TiO}_{2}{ }^{20,22}$ and $\mathrm{Pd} / \mathrm{TiO}_{2}$ junctions ${ }^{59}$. Conversely, when IR light is considered, light is not absorbed by the $\mathrm{TiO}_{2}$ layer and electrons are exited at the $n$-type Si-NPls while generating a flow of electrons towards the surface and to the $\mathrm{TiO}_{2}(\mathrm{HER})$. This synergistic effect 
was proposed by Tao $\mathrm{Ji}$ et $a .^{52}$ and takes into account the dual nature of light radiation absorption, IR and UV, on similar systems. For the case presented here, the addition of nanostructured Pd layer allows for the additional generation of hot carriers at the Pd surface. ${ }^{60}$ Hot carriers are generated by SPR, and in the presence of a schottky barrier, allow the faster separation of carriers and longer lifetime of catalytically active centers (HER). Therefore, the general mechanism can be illustrated as presented in Figure $3 \mathrm{c}$ where the full electromagnetic spectrum of the sun is considered. The IR wavelengths activate electrons in $n$-type Si-NPls ( 1000 nm), medium wavelengths generate hot-carriers by surface plasmon resonance (SPR), $(600-860 \mathrm{~nm})(\text { Figure } 2 \mathrm{c})^{55}$ and the UV part of the spectra generates electrons in the $\mathrm{TiO}_{2}$ layer $(<500 \mathrm{~nm})$. In zero bias conditions, like in a submerged sample, the flow of electrons is directed to the surface, where generated hot carriers promote HER on the Pd surface while changing its work function and pushing the electron flow further towards HER. Moreover, since oxygen is present in the solution, hydrogen should be released as water vapor, while oxygen deprived regions should generate $\mathrm{H}_{2}$ gas. Nevertheless, in the case of degradation of contaminants, the continuous injection of electrons will drive the degradation of rhodamine as shown in the literature. ${ }^{61}$ Finally, the generation of gas bubbles increases the absorption of IR light and heat like in a small scale greenhouse effect, which overall increases the water evaporation efficiency of the system.

\section{Conclusions}

To conclude, in this study we have successfully prepared conformal $\mathrm{Pd}$ coating on $\mathrm{TiO}_{2} / \mathrm{Si}$ NPls by ALD. The resulting structures show superior mechanical resilience than those of pure SiNPls, which expands their applicability in mechanical demanding environments. The Pd coating slightly diminishes the photoactivity towards OER of the nanostructures due to the schottky contacts generated by the $\mathrm{Pd} / \mathrm{TiO}_{2}$ nanodiodes. Nevertheless, in zero bias conditions, the absorption of selected parts of the solar spectrum by each element of heterojunction namely UV spectra by $\mathrm{TiO}_{2}$, near infrared by Pd particles in the shape of SPR generated excitons, and medium far infrared by $n$-type Si-NPls, promotes an electron flux that drives the reaction towards HER on the nanostructures. The combination of these factors make these nanostructures highly applicable in solar driven water remediation and sun absorption boosted by HER, while 
remaining competitive in biased photocatalysis towards OER. Further studies are needed in order to determine the general limit of this synergistic architecture. Finally, further studies on thermal stability, $\mathrm{Pd}$ binding on $\mathrm{TiO}_{2}$ surfaces and the influence of $\mathrm{Pd}$ thickness would definitely optimize the architecture here proposed, the presented methodology shows the clear applicability of Schottky nanodiodes and tunability of the OER/HER reaction on single structures for wide solar spectra water remediation.

\section{Experimental}

Fabrication and Nanostructuring: Commercially available n-type (100) (phosphorus doped, 0.01-0.02 $\Omega \cdot \mathrm{cm}$ ) polished silicon wafers were used in this study. SiNPls were prepared by a method similar to our previous papers. ${ }^{6,10}$ Briefly, Si-NPls were fabricated by combining nanosphere lithography (NSL), reactive ion etching (RIE) and metal-assisted chemical etching (MACE). Silicon samples were cleaned by ethanol, acetone, and dionized water (DI). Polystyrene nanosphere $10 \%$ solution (mean diameter $800 \mathrm{~nm}$ ) was mixed with an equal amount of ethanol and then applied to the deposition of a monolayer mask on a silicon surface by spin-coating technique. Then, the size of nanosphere was decreased using (RIE) (MicroSys 200) in oxygen plasma (RF power - $70 \mathrm{~W}$, pressure - 0.6 mbar). After the $50 \mathrm{~nm}$ layer gold deposition (magnetron sputtering machine Quorum Q160T), the samples were etched in aqueous solution containing $\mathrm{HF}(40 \%), \mathrm{H}_{2} \mathrm{O}_{2}(30 \%)$, and ultrapure $\mathrm{H}_{2} \mathrm{O}$ at a ratio of 80:80:20, respectively. The $\mathrm{Au}$ layer was removed using a solution of Aqua Regia $\left(\mathrm{HCl}(35 \%)\right.$ and $\mathrm{HNO}_{3}(65 \%)$ at ratio $\left.1: 2\right)$ and then samples were dipped in HF (5\%) solution for $5 \mathrm{~min}$ to remove native oxide, cleaned by DI and blown dry with $\mathrm{N}_{2}$. After nanopillars fabrication, the ALD $\mathrm{TiO}_{2}$ layer (400 ALD cycles) was deposited using $\mathrm{TiCl}_{4}$ and water as ALD precursors at $200^{\circ} \mathrm{C}$. The growth rate was typically $0.45 \AA$ A/cycle on the planar Si surface. The ALD cycle consisted of $0.5 \mathrm{~s}$ exposure to $\mathrm{TiCl}_{4}, 8 \mathrm{~s} \mathrm{~N} 2$ purge, $0.5 \mathrm{~s}$ exposure to water and $8 \mathrm{~s} \mathrm{~N}_{2}$ purge. ALD of $\mathrm{Pd}$ was based on $\mathrm{Pd}(\mathrm{hfac})_{2}$ and formalin. The conformal Pd coating was achieved by applying $200 \mathrm{ALD}$ at $220^{\circ} \mathrm{C}$. The typical ALD cycle consisted of $5 \mathrm{~s}$ pulse of $\mathrm{Pd}(\mathrm{hfac})_{2}, 15 \mathrm{~s}$ exposure, and $10 \mathrm{~s}$ purge, followed by a $1 \mathrm{~s}$ pulse of formalin, $15 \mathrm{~s}$ exposure, and $60 \mathrm{~s}$ purge with Ar. The bubbler containing the $\mathrm{Pd}(\mathrm{hfac})_{2}$ precursor was heated at $70{ }^{\circ} \mathrm{C}$, and the formalin container was kept at room temperature. 
Physico-Chemical Characterization: Samples were investigated by Grazing Incident X-ray spectrometry (GI-XRD) in a X'pert ${ }^{3}(\mathrm{XL})$ MRD PANalytical, working in a $40 \mathrm{kv} 45 \mathrm{~mA}$ and $\mathrm{Cu}$ $\mathrm{K} \alpha(\lambda=1.54 \AA$ A). Scanning Electron Microscopy (SEM) in a JEOL JSMM7001F microscope. High Resolution Electron Microscopy (HR-TEM), in a JEOL ARM 200F microscope working at 200 $\mathrm{kV}$, equipped with an Energy Dispersive X-ray Spectroscopy (EDX). Samples were cut for examination using a Focus Ion Beam (FIB) system by JEOL-JIB-4000. Nanoindentation experiments were performed in a Triboindenter TI-950 by Hysitron, methodology and other technical parameters were described in detail elsewhere. ${ }^{6,10,19,62}$ Optical response was studied in UV-Vis spectrophometer Lambda 950UV/VIS/NR with $1 \mathrm{~nm}$ step. Finally, the diffuse reflectance was measure in an Ocean Optics QE PRO spectrometer coupled with an integrating sphere.

Electrochemical Characterization: Characterization of working electrodes was performed using the potentionstat-galvanostat system AutoLab PGStat $302 \mathrm{~N}$ in a standard three-electrode assembly at room temperature. Counter electrode was $\mathrm{Pt}$ gauze and reference electrode was $\mathrm{Ag} / \mathrm{AgCl} / 0.1 \mathrm{M} \mathrm{KCl}$. The electrolyte $(180 \mathrm{~mL})$ of $0.5 \mathrm{M} \mathrm{K}_{2} \mathrm{SO}_{4}(\mathrm{pH}=7)$, was flushed with argon gas $(99.999 \%$ purity), for 40 minutes before measurements. The samples were irradiated with a solar simulator (LOT Orietl) equipped with an AM 1.5G filter allowing to obtain full spectrum UV-vis light. The irradiance of incident light was calibrated to $100 \mathrm{~mW} / \mathrm{cm}^{2}$ using a reference cell ( $\mathrm{Si}$ solar cell calibrated for air mass $1.5 \mathrm{G} 100 \mathrm{~W} / \mathrm{m}^{2} @ 25^{\circ} \mathrm{C}$, Rera). Electrochemical impedance spectroscopy (EIS), in dark and UV-vis conditions, were performed in the frequency range: $0.1 \mathrm{~Hz}-20 \mathrm{kHz}$, at the amplitude of $10 \mathrm{mV}$ and open circuit voltage as the potential of working electrode, EIS data was analyzed and fitted using the electrochemical equivalent circuit (EEQC) in Z-View software, using a Constant Phase Elements in parallel with a resistor (R-CPE) for $\mathrm{TiO}_{2} / \mathrm{Pd}$ and $\mathrm{TiO}_{2}$ in dark, while two (2) $\mathrm{R}-\mathrm{CPE}$ circuits in series were used for the $\mathrm{TiO}_{2}$ under illumination ${ }^{63,64}$. The elements fitted showed a goodness-of-fit-value $\left(\chi^{2}\right)$ below $\sim 10^{-4}$. Cyclic voltammetry (dark conditions) potential range: from -0.6 to $+0.6 \mathrm{~V}$ vs. $\mathrm{Ag} / \mathrm{AgCl} / 0.1 \mathrm{M}$ $\mathrm{KCl}$ at a scanning speed of $50 \mathrm{mV} / \mathrm{s}$. Linear voltamperometry (dark and UV-vis conditions) curves were recorded from -0.7 to $+1.2 \mathrm{~V}$ vs. $\mathrm{Ag} / \mathrm{AgCl} / 0.1 \mathrm{M} \mathrm{KCl}$ at the scanning speed of 10 $\mathrm{mV} / \mathrm{s}$. Finally, chronoamperometry (dark/UV-vis for a change) were measured in polarization conditions: $+1.0 \mathrm{~V}$ vs. $\mathrm{Ag} / \mathrm{AgCl} / 0.1 \mathrm{M} \mathrm{KCl}$ with irradiation/dark periods of $20 \mathrm{~s}$ 
Heat Absorption and Photodegradation: Heat absorption experiments were performed by placing a $1 \times 0.5 \mathrm{~cm}$ sample in an optically graded quartz cuvette and adding 10 milliliters of deionized water (Milli-Q water $18.2 \mathrm{M} \Omega \mathrm{cm}$ ). Samples were placed in a vertical position against the back window of the cuvette in order to collect the entire incident light at a $90^{\circ}$ angle. Solar simulator (Photon Institute - Poland) was focused on the samples to reach 1 Sun illumination $\left(100 \mathrm{mWcm}^{-}\right.$ ${ }^{2}$ ) by using a calibrated silicon solar cell (15151, ABET). Cuvettes were rinse with plenty of deionized water after experiment and fresh water was used for new experiments (room temperature $\sim 23^{\circ}$ ). Water temperature was monitored by a thermocouple in contact with the water slightly above the incident light. Water evaporation was continuously measured by a microbalance in which the cuvette was suspended by a holder (see Figure S7).

Photodegradation experiments were evaluated using rhodamine B as a model organic dye. The concentration of rhodamine B solution was $5 \times 10^{-2} \mathrm{mg} / \mathrm{mL}$. Before irradiation, the samples $(1 \mathrm{x} 0.5$ $\mathrm{cm}^{2}$ ) were dipped in the solution and then stirred in the darkness for 30 min to reach adsorptiondesorption equilibrium. The UV-vis light irradiation was carried out in a quartz cuvette using Xe lamp (Lambda LS model LB-LS/OF 30R, 4.5 Watts/300W) at distant of $30 \mathrm{~cm}$ from the surface of solution in horizontal geometry. Then the absorption spectrum was constantly recorded by the Ocean Optics USB spectrometer (QE65-PRO). The remaining concentration of rhodamine B in the solution was estimated using absorbance spectrum at $\lambda=550 \mathrm{~nm}$. The calibration curve of absorbance was used to estimate the photocatalytic activity of the samples.

\section{Supplementary Material:}

Supplementary are available online at the Journal's homepage. The material include additional SEM micrographs (Figure S1), EDX mapping (Figure S2), Load vs Displacement experiment on Si-NPs (Figure S3), Silicon Absorption (Figure S4), Stability (Figure S5), Temperature recorded on the water evaporation experiments (Figure S6) and Schematic and pictures of the Water Evaporation set-up. (Figure S7)

\section{Acknowledgements}

I.I. acknowledges the financial support from the National Science Centre of Poland by the SONATA 11 project UMO-2016/21/D/ST3/00962. M.P. acknowledges the financial support from the National Science Centre (NCN) of Poland by the PRELUDIUM 12 project UMO- 
2016/23/N/ST3/01356 and the National Centre for Research and Development (NCBR) by the project POWR.03.02.00-00-I032/16. K.S. acknowledges the financial support from the National Science Centre (NCN) of Poland by Sonata-BIS no 2017/26/E/ST5/00416. E.C, M.W, M.B and I.I acknowledge the support of RISE- H2020-EU.1.3.3. grant (ID:778157) CanBioSe. Finally, authors acknowledge the support from Prof. Stefan Jurga (CNBM-AMU).

\section{Author Contributions:}

All authors have given approval to the final version of the manuscript.

\section{References}

(1) Haydous, F.; Döbeli, M.; Si, W.; Waag, F.; Li, F.; Pomjakushina, E.; Wokaun, A.; Gökce, B.; Pergolesi, D.; Lippert, T. Oxynitride Thin Films versus Particle-Based Photoanodes: A Comparative Study for Photoelectrochemical Solar Water Splitting. ACS Appl. Energy Mater. 2019, 2 (1), 754-763.

(2) Hisatomi, T.; Domen, K. Reaction Systems for Solar Hydrogen Production via Water Splitting with Particulate Semiconductor Photocatalysts. Nat. Catal. 2019, 2 (5), 387-399.

(3) Wang, Z.; Li, C.; Domen, K. Recent Developments in Heterogeneous Photocatalysts for Solar-Driven Overall Water Splitting. Chem. Soc. Rev. 2019, 48 (7), 2109-2125.

(4) Abe, J. O.; Popoola, A. P. I.; Ajenifuja, E.; Popoola, O. M. Hydrogen Energy, Economy and Storage: Review and Recommendation. Int. J. Hydrogen Energy 2019, 44 (29), 15072-15086.

(5) Kertmen, A.; Barbé, E.; Szkoda, M.; Siuzdak, K.; Babačić, V.; Torruella, P.; Iatsunskyi, I.; Kotkowiak, M.; Rytel, K.; Estradé, S.; Peiró, F.; Jurga, S.; Li, Y.; Coy, E. Photoelectrochemically Active N-Adsorbing Ultrathin TiO 2 Layers for Water-Splitting Applications Prepared by Pyrolysis of Oleic Acid on Iron Oxide Nanoparticle Surfaces under Nitrogen Environment. Adv. Mater. Interfaces 2018, 1801286.

(6) Pavlenko, M.; Siuzdak, K.; Coy, E.; Jancelewicz, M.; Jurga, S.; Iatsunskyi, I. Silicon/TiO 2 Core-Shell Nanopillar Photoanodes for Enhanced Photoelectrochemical Water Oxidation. Int. J. Hydrogen Energy 2017, 42 (51), 30076-30085.

(7) Kang, Z.; Si, H.; Zhang, S.; Wu, J.; Sun, Y.; Liao, Q.; Zhang, Z.; Zhang, Y. Interface Engineering for Modulation of Charge Carrier Behavior in ZnO Photoelectrochemical Water Splitting. Adv. Funct. Mater. 2019, 29 (15), 1808032.

(8) Chai, X.; Zhang, H.; Pan, Q.; Bian, J.; Chen, Z.; Cheng, C. 3D Ordered Urchin-like TiO2@Fe2O3 Arrays Photoanode for Efficient Photoelectrochemical Water Splitting. Appl. Surf. Sci. 2019, 470, 668-676.

(9) Huang, C.-L.; Chuah, X.-F.; Hsieh, C.-T.; Lu, S.-Y. NiFe Alloy Nanotube Arrays as Highly Efficient Bifunctional Electrocatalysts for Overall Water Splitting at High Current Densities. ACS Appl. Mater. Interfaces 2019, 11 (27), 24096-24106.

(10) Pavlenko, M.; Coy, E. L.; Jancelewicz, M.; Załęski, K.; Smyntyna, V.; Jurga, S.; Iatsunskyi, I. Enhancement of Optical and Mechanical Properties of Si Nanopillars by ALD TiO 2 Coating. RSC Adv. 2016, 6 (99), 97070-97076.

(11) Lv, C.; Chen, Z.; Chen, Z.; Zhang, B.; Qin, Y.; Huang, Z.; Zhang, C. Silicon Nanowires 
Loaded with Iron Phosphide for Effective Solar-Driven Hydrogen Production. J. Mater. Chem. A 2015, 3 (34), 17669-17675.

(12) Dytrych, P.; Drinek, V.; Bumba, J.; Kastanek, F.; Solcova, O. Silicon Nanowires' Based Photoanode for Hydrogen Evolution. Int. J. Hydrogen Energy 2018, 43 (39), 1813618141.

(13) Cai, Q.; Hong, W.; Jian, C.; Li, J.; Liu, W. Insulator Layer Engineering toward Stable Si Photoanode for Efficient Water Oxidation. ACS Catal. 2018, 8 (10), 9238-9244.

(14) Yu, Y.; Zhang, Z.; Yin, X.; Kvit, A.; Liao, Q.; Kang, Z.; Yan, X.; Zhang, Y.; Wang, X. Enhanced Photoelectrochemical Efficiency and Stability Using a Conformal TiO2 Film on a Black Silicon Photoanode. Nat. Energy 2017, 2 (6), 17045.

(15) Scheuermann, A. G.; Lawrence, J. P.; Meng, A. C.; Tang, K.; Hendricks, O. L.; Chidsey, C. E. D.; McIntyre, P. C. Titanium Oxide Crystallization and Interface Defect Passivation for High Performance Insulator-Protected Schottky Junction MIS Photoanodes. ACS Appl. Mater. Interfaces 2016, 8 (23), 14596-14603.

(16) Hwang, Y. J.; Boukai, A.; Yang, P. High Density N-Si/n-TiO 2 Core/Shell Nanowire Arrays with Enhanced Photoactivity. Nano Lett. 2009, 9 (1), 410-415.

(17) Ros, C.; Andreu, T.; Hernández-Alonso, M. D.; Penelas-Pérez, G.; Arbiol, J.; Morante, J. R. Charge Transfer Characterization of ALD-Grown TiO 2 Protective Layers in Silicon Photocathodes. ACS Appl. Mater. Interfaces 2017, 9 (21), 17932-17941.

(18) Zhang, F.-Q.; Hu, Y.; Sun, R.-N.; Fu, H.; Peng, K.-Q. Gold-Sensitized Silicon/ZnO Core/Shell Nanowire Array for Solar Water Splitting. Front. Chem. 2019, 7, 206.

(19) Robak, E.; Coy, E.; Kotkowiak, M.; Jurga, S.; Załęski, K.; Drozdowski, H. The Effect of $\mathrm{Cu}$ Doping on the Mechanical and Optical Properties of Zinc Oxide Nanowires Synthesized by Hydrothermal Route. Nanotechnology 2016, 27 (17), 175706.

(20) Cheng, L.; Zhang, D.; Liao, Y.; Li, F.; Zhang, H.; Xiang, Q. Constructing Functionalized Plasmonic Gold/Titanium Dioxide Nanosheets with Small Gold Nanoparticles for Efficient Photocatalytic Hydrogen Evolution. J. Colloid Interface Sci. 2019, 555, 94-103.

(21) Li, D.; Wang, C.; Strmcnik, D. S.; Tripkovic, D. V.; Sun, X.; Kang, Y.; Chi, M.; Snyder, J. D.; van der Vliet, D.; Tsai, Y.; Stamenkovic, V. R.; Sun, S.; Markovic, N. M. Functional Links between Pt Single Crystal Morphology and Nanoparticles with Different Size and Shape: The Oxygen Reduction Reaction Case. Energy Environ. Sci. 2014, 7 (12), 40614069.

(22) Luna, M.; Gatica, J. M.; Vidal, H.; Mosquera, M. J. Au-TiO2/SiO2 Photocatalysts with NOx Depolluting Activity: Influence of Gold Particle Size and Loading. Chem. Eng. J. 2019, 368, 417-427.

(23) Yang, W.; Xiong, Y.; Zou, L.; Zou, Z.; Li, D.; Mi, Q.; Wang, Y.; Yang, H. Plasmonic Pd Nanoparticle- and Plasmonic Pd Nanorod-Decorated BiVO4 Electrodes with Enhanced Photoelectrochemical Water Splitting Efficiency Across Visible-NIR Region. Nanoscale Res. Lett. 2016, 11 (1), 283.

(24) Hong, W.; Cai, Q.; Ban, R.; He, X.; Jian, C.; Li, J.; Li, J.; Liu, W. High-Performance Silicon Photoanode Enhanced by Gold Nanoparticles for Efficient Water Oxidation. ACS Appl. Mater. Interfaces 2018, 10 (7), 6262-6268.

(25) Tang-Kong, R.; Winter, R.; Brock, R.; Tracy, J.; Eizenberg, M.; Dauskardt, R. H.; McIntyre, P. C. The Role of Catalyst Adhesion in ALD-TiO 2 Protection of Water Splitting Silicon Anodes. ACS Appl. Mater. Interfaces 2018, 10 (43), 37103-37109.

(26) Wang, A.; Zhao, L.; Yu, J.; Zhou, W.; Chu, B.; Liu, H. Co Nanoparticles@ N-Doped Carbon Coated on Carbon Nanotube@Defective Silica as Non-Noble Photocathode for 
Efficient Photoelectrochemical Hydrogen Generation. Int. J. Hydrogen Energy 2018, 43 (19), 9279-9286.

(27) Zhou, W.; Guan, Y.; Wang, D.; Zhang, X.; Liu, D.; Jiang, H.; Wang, J.; Liu, X.; Liu, H.; Chen, S. PdO/TiO 2 and $\mathrm{Pd} / \mathrm{TiO} 2$ Heterostructured Nanobelts with Enhanced Photocatalytic Activity. Chem. - An Asian J. 2014, 9 (6), 1648-1654.

(28) Zakaria, M. B.; Malgras, V.; Nagata, T.; Kim, J.; Bando, Y.; Fatehmulla, A.; Aldhafiri, A. M.; Farooq, W. A.; Jikihara, Y.; Nakayama, T.; Yamauchi, Y.; Lin, J. Gold Nanoparticles Anchored on Mesoporous Zirconia Thin Films for Efficient Catalytic Oxidation of Carbon Monoxide at Low Temperatures. Microporous Mesoporous Mater. 2019, 288, 109530.

(29) Silina, Y. E.; Koch, M.; Herbeck-Engel, P.; Iatsunskyi, I. Exploring the Potential of High Resolution Inductively Coupled Plasma Mass Spectrometry towards Non-Destructive Control and Validation of Electroless Gold Nanoparticles onto Silicon Nanowires Hybrids. Anal. Methods 2019.

(30) Rao, X.; Guyon, C.; Ognier, S.; Da Silva, B.; Chu, C.; Tatoulian, M.; Hassan, A. A. High Density Gold Nanoparticles Immobilized on Surface via Plasma Deposited APTES Film for Decomposing Organic Compounds in Microchannels. Appl. Surf. Sci. 2018, 439, 272281.

(31) Weber, M. J.; Verheijen, M. A.; Bol, A. A.; Kessels, W. M. M. Sub-Nanometer Dimensions Control of Core/Shell Nanoparticles Prepared by Atomic Layer Deposition. Nanotechnology 2015, 26 (9), 094002.

(32) Leskelä, M.; Ritala, M. Atomic Layer Deposition Chemistry: Recent Developments and Future Challenges. Angew. Chemie Int. Ed. 2003, 42 (45), 5548-5554.

(33) Weber, M.; Julbe, A.; Ayral, A.; Miele, P.; Bechelany, M. Atomic Layer Deposition for Membranes: Basics, Challenges, and Opportunities. Chem. Mater. 2018, 30 (21), 7368 7390 .

(34) Weber, M.; Tuleushova, N.; Zgheib, J.; Lamboux, C.; Iatsunskyi, I.; Coy, E.; Flaud, V.; Tingry, S.; Cornu, D.; Miele, P.; Bechelany, M.; Holade, Y. Enhanced Electrocatalytic Performance Triggered by Atomically Bridged Boron Nitride between Palladium Nanoparticles and Carbon Fibers in Gas-Diffusion Electrodes. Appl. Catal. B Environ. 2019, 257, 117917.

(35) Weber, M.; Kim, J.-Y.; Lee, J.-H.; Kim, J.-H.; Iatsunskyi, I.; Coy, E.; Miele, P.; Bechelany, M.; Kim, S. S. Highly Efficient Hydrogen Sensors Based on Pd Nanoparticles Supported on Boron Nitride Coated ZnO Nanowires. J. Mater. Chem. A 2019, 7 (14), 8107-8116.

(36) Weber, M.; Kim, J.-H.; Lee, J.-H.; Kim, J.-Y.; Iatsunskyi, I.; Coy, E.; Drobek, M.; Julbe, A.; Bechelany, M.; Kim, S. S. High-Performance Nanowire Hydrogen Sensors by Exploiting the Synergistic Effect of Pd Nanoparticles and Metal-Organic Framework Membranes. ACS Appl. Mater. Interfaces 2018, 10 (40), 34765-34773.

(37) Merenda, A.; Weber, M.; Bechelany, M.; Allioux, F.-M.; Hyde, L.; Kong, L.; Dumée, L. F. Fabrication of Pd-TiO2 Nanotube Photoactive Junctions via Atomic Layer Deposition for Persistent Pesticide Pollutants Degradation. Appl. Surf. Sci. 2019, 483, 219-230.

(38) Gao, M.; Zhu, L.; Peh, C. K.; Ho, G. W. Solar Absorber Material and System Designs for Photothermal Water Vaporization towards Clean Water and Energy Production. Energy and Environmental Science. 2019.

(39) Zhu, L.; Ding, T.; Gao, M.; Peh, C. K. N.; Ho, G. W. Shape Conformal and Thermal Insulative Organic Solar Absorber Sponge for Photothermal Water Evaporation and Thermoelectric Power Generation. Adv. Energy Mater. 2019, 9 (22), 1900250. 
(40) Zhu, L.; Gao, M.; Peh, C. K. N.; Ho, G. W. Solar-Driven Photothermal Nanostructured Materials Designs and Prerequisites for Evaporation and Catalysis Applications. Materials Horizons. 2018.

(41) Cai, J.; Qi, L. Recent Advances in Antireflective Surfaces Based on Nanostructure Arrays. Mater. Horizons 2015, 2 (1), 37-53.

(42) Asapu, R.; Claes, N.; Ciocarlan, R.-G.; Minjauw, M.; Detavernier, C.; Cool, P.; Bals, S.; Verbruggen, S. W. Electron Transfer and Near-Field Mechanisms in Plasmonic GoldNanoparticle-Modified TiO2 Photocatalytic Systems. ACS Appl. Nano Mater. 2019, 2 (7), 4067-4074.

(43) Zolotavin, P.; Alabastri, A.; Nordlander, P.; Natelson, D. Plasmonic Heating in Au Nanowires at Low Temperatures: The Role of Thermal Boundary Resistance. ACS Nano 2016, 10 (7), 6972-6979.

(44) Huang, J.; He, Y.; Wang, L.; Huang, Y.; Jiang, B. Bifunctional Au@TiO2 Core-Shell Nanoparticle Films for Clean Water Generation by Photocatalysis and Solar Evaporation. Energy Convers. Manag. 2017, 132, 452-459.

(45) Bellet, D.; Lamagnère, P.; Vincent, A.; Bréchet, Y. Nanoindentation Investigation of the Young's Modulus of Porous Silicon. J. Appl. Phys. 1996, 80 (7), 3772-3776.

(46) Brytavskyi, I.; Hušeková, K.; Myndrul, V.; Pavlenko, M.; Coy, E.; Zaleski, K.; Gregušová, D.; Yate, L.; Smyntyna, V.; Iatsunskyi, I. Effect of Porous Silicon Substrate on Structural, Mechanical and Optical Properties of MOCVD and ALD Ruthenium Oxide Nanolayers. Appl. Surf. Sci. 2019, 471, 686-693.

(47) Sarkar, S.; Peter, S. C. An Overview on Pd-Based Electrocatalysts for the Hydrogen Evolution Reaction. Inorg. Chem. Front. 2018, 5 (9), 2060-2080.

(48) Mehrdad, A.; Massoumi, B.; Hashemzadeh, R. Kinetic Study of Degradation of Rhodamine B in the Presence of Hydrogen Peroxide and Some Metal Oxide. Chem. Eng. J. 2011, 168 (3), 1073-1078.

(49) Wilhelm, P.; Stephan, D. Photodegradation of Rhodamine B in Aqueous Solution via SiO2@TiO2 Nano-Spheres. J. Photochem. Photobiol. A Chem. 2007, 185 (1), 19-25.

(50) Barakat, N. A. M.; Kanjwal, M. A.; Chronakis, I. S.; Kim, H. Y. Influence of Temperature on the Photodegradation Process Using Ag-Doped TiO2 Nanostructures: Negative Impact with the Nanofibers. J. Mol. Catal. A Chem. 2013, 366, 333-340.

(51) Chiu, Y.-H.; Chang, T.-F. M.; Chen, C.-Y.; Sone, M.; Hsu, Y.-J. Mechanistic Insights into Photodegradation of Organic Dyes Using Heterostructure Photocatalysts. Catalysts 2019, 9 (5), 430.

(52) Ji, T.; Cui, Z.; Zhang, W.; Cao, Y.; Zhang, Y.; He, S.; Xu, M.; Sun, Y.; Zou, R.; Hu, J. UV and Visible Light Synergetic Photodegradation Using Rutile TiO 2 Nanorod Arrays Based on a p-n Junction. Dalt. Trans. 2017, 46 (13), 4296-4302.

(53) Wu, J.; Lu, S.; Ge, D.; Zhang, L.; Chen, W.; Gu, H. Photocatalytic Properties of Pd/TiO 2 Nanosheets for Hydrogen Evolution from Water Splitting. RSC Adv. 2016, 6 (72), 6750267508.

(54) Yamamoto, N.; Tonomura, S.; Matsuoka, T.; Tsubomura, H. A Study on a PalladiumTitanium Oxide Schottky Diode as a Detector for Gaseous Components. Surf. Sci. 1980, 92 (2-3), 400-406.

(55) Tittl, A.; Mai, P.; Taubert, R.; Dregely, D.; Liu, N.; Giessen, H. Palladium-Based Plasmonic Perfect Absorber in the Visible Wavelength Range and Its Application to Hydrogen Sensing. Nano Lett. 2011, 11 (10), 4366-4369.

(56) Ji, X. Z.; Somorjai, G. A. Continuous Hot Electron Generation in Pt/TiO 2 , Pd/TiO 2 , and 
Pt/GaN Catalytic Nanodiodes from Oxidation of Carbon Monoxide. J. Phys. Chem. B 2005, 109 (47), 22530-22535.

(57) Khan, M. R.; Chuan, T. W.; Yousuf, A.; Chowdhury, M. N. K.; Cheng, C. K. Schottky Barrier and Surface Plasmonic Resonance Phenomena towards the Photocatalytic Reaction: Study of Their Mechanisms to Enhance Photocatalytic Activity. Catal. Sci. Technol. 2015, 5 (5), 2522-2531.

(58) Nosaka, Y.; Komori, S.; Yawata, K.; Hirakawa, T.; Nosaka, A. Y. Photocatalytic 'OH Radical Formation in TiO 2 Aqueous Suspension Studied by Several Detection Methods. Phys. Chem. Chem. Phys. 2003, 5 (20), 4731-4735.

(59) Zhang, H.; Meng, D.; Fu, B.; Fan, H.; Cai, R.; Fu, P. P.; Wu, X. Separation of Charge Carriers and Generation of Reactive Oxygen Species by TiO 2 Nanoparticles Mixed with Differently-Coated Gold Nanorods under Light Irradiation. J. Environ. Sci. Heal. Part C 2019, 37 (2), 81-98.

(60) Robatjazi, H.; Bahauddin, S. M.; Doiron, C.; Thomann, I. Direct Plasmon-Driven Photoelectrocatalysis. Nano Lett. 2015, 15 (9), 6155-6161.

(61) AlHamedi, F. H.; Rauf, M. A.; Ashraf, S. S. Degradation Studies of Rhodamine B in the Presence of UV/H2O2. Desalination 2009, 239 (1-3), 159-166.

(62) Coy, E.; Yate, L.; Kabacińska, Z.; Jancelewicz, M.; Jurga, S.; Iatsunskyi, I. Topographic Reconstruction and Mechanical Analysis of Atomic Layer Deposited Al 2 O 3 /TiO 2 Nanolaminates by Nanoindentation. Mater. Des. 2016, 111, 584-591.

(63) Orazem, M. E.; Tribollet, B. Electrochemical Impedance Spectroscopy; John Wiley \& Sons, Inc.: Hoboken, NJ, USA, 2008.

(64) Lasia, A. Electrochemical Impedance Spectroscopy and Its Applications. In Modern Aspects of Electrochemistry; Kluwer Academic Publishers: Boston; pp 143-248. 